\title{
On sumsets of multisets in $\mathbb{Z}_{p}^{m}$
}

\author{
Kaisa Matomäki* \\ Department of Mathematics \\ University of Turku \\ 20014 Turku, Finland \\ ksmato@utu.fi
}

Submitted: Apr 5, 2013; Accepted: Aug 20, 2013; Published: Aug 30, 2013

Mathematics Subject Classifications: 11B13

\begin{abstract}
For a sequence $A$ of given length $n$ contained in $\mathbb{Z}_{p}^{2}$ we study how many distinct subsums $A$ must have when $A$ is not "wasteful" by containing too many elements in same subgroup. Martin, Peilloux and Wong have made a conjecture for a sharp lower bound and established it when $n$ is not too large whereas Peng has previously established the conjecture for large $n$. In this note we build on these earlier works and add an elementary argument leading to the conjecture for every $n$.

Martin, Peilloux and Wong also made a more general conjecture for sequences in $\mathbb{Z}_{p}^{m}$. Here we show that the special case $n=m p-1$ of this conjecture implies the whole conjecture and that the conjecture is equivalent to a strong version of the additive basis conjecture of Jaeger, Linial, Payan and Tarsi.
\end{abstract}

\section{Introduction}

For a sequence $A$ contained in an abelian group $\mathbf{G}$ we write $\sum A$ for the set of all subsums of $A$, that is, for $A=\left(a_{1}, \ldots, a_{n}\right)$,

$$
\sum A=\left\{\sum_{i \in I} a_{i}: I \subseteq\{1, \ldots, n\}\right\} .
$$

Note that $\sum A$ always contains 0 , the sum of an empty sequence. As the order of the elements of $A$ is not relevant here, we will from now on think of $A$ as a multiset. For a set or multiset $B$, we write $|B|$ for the cardinality of $B$, counted with multiplicity, and $\# B$ for the cardinality of $B$ counted without multiplicity.

${ }^{*}$ Supported by the Academy of Finland grant no. 137883 
Here we are interested in the relationship between $|A|$ and $\# \sum A$. As pointed out for instance in [3, Lemma 1.3], in case $\mathbf{G}=\mathbb{Z}_{p}$ one gets the following result easily by multiple applications of the Cauchy-Davenport inequality (see [6, Theorem 5.4]).

Lemma 1. Let $p \in \mathbb{P}$ and let $A$ be a multiset contained in $\mathbb{Z}_{p}^{*}$. Then

$$
\# \sum A \geqslant \min \{p,|A|+1\}
$$

This lower bound is sharp as $A$ may consist of $|A|$ copies of a single element.

Let us now consider the case $\mathbf{G}=\mathbb{Z}_{p}^{2}$. In this case one might not get a better lower bound than the above if much of $A$ is contained in a single subgroup. In particular it is "wasteful" for $A$ to contain more than $p-1$ elements from any subgroup since by Lemma 1 already $p-1$ elements guarantee that $\sum A$ contains the whole subgroup. In light of this we make the following definition (following [3]).

Definition 2. A multiset $A$ contained in $\mathbb{Z}_{p}^{2}$ is called valid if $0 \notin A$ and every non-trivial subgroup of $\mathbb{Z}_{p}^{2}$ contains at most $p-1$ points of $A$ (counting multiplicity).

For a valid multiset $A$ in $\mathbb{Z}_{p}^{2}$ with at most $p-1$ elements, one has again the sharp lower bound $\# \sum A \geqslant|A|+1$. On the other hand, for large multisets Peng [4] has shown the following.

Theorem 3. Let $p \in \mathbb{P}$ and let $A$ be a valid multiset contained in $\mathbb{Z}_{p}^{2}$ with $|A| \geqslant 2 p-1$. Then $\sum A=\mathbb{Z}_{p}^{2}$.

Hence we can concentrate on the case $p \leqslant|A| \leqslant 2 p-2$. Martin, Peilloux and Wong [3] have made the following conjecture.

Conjecture 4. Let $p \in \mathbb{P}$, let $k$ be a non-negative integer, and let $A$ be a valid multiset contained in $\mathbb{Z}_{p}^{2}$ with $|A|=p+k$. If $k \leqslant p-3$, then $\# \sum A \geqslant(k+2) p$ and if $k=p-2$, then $\# \sum A \geqslant p^{2}-1$.

If true, this conjecture would be sharp as pointed out in [3]: First, for $k \leqslant p-3$, the multiset $A$ may consist of $p-1$ copies of $(1,0)$ and $k+1$ copies of $(0,1)$, so that $\sum A=\mathbb{Z}_{p} \times\{0, \ldots, k+1\}$. Second, for $k=p-2, A$ may consist of $p-2$ copies of $(1,0)$ and one copy of each $(i, 1), 0 \leqslant i \leqslant p-1$, so that $\sum A=\mathbb{Z}_{p}^{2} \backslash\{(p-1,0)\}$.

Martin, Peilloux and Wong [3] proved the conjecture when

$$
k \leqslant \max \{1, \sqrt{p /(2 \log p+1)}-1\} .
$$

Here we will prove the conjecture for every $k$.

Theorem 5. Conjecture 4 holds.

Martin, Peilloux and Wong [3] also generalised Conjecture 4 to $\mathbb{Z}_{p}^{m}$ for $m \geqslant 2$. They again want to avoid "wasteful" sets and thus only consider "valid" sets. To easily define validity in this setting, for a subgroup $\mathbf{H}$ of $\mathbb{Z}_{p}^{m}$, we write $\operatorname{dim} \mathbf{H}=d$ where $d$ is the integer for which $\mathbf{H}$ is isomorphic to $\mathbb{Z}_{p}^{d}$. 
Definition 6. Let $m \geqslant 2$. A multiset $A$ contained in $\mathbb{Z}_{p}^{m}$ is called valid if $0 \notin A$ and every non-trivial subgroup $\mathbf{H}$ of $\mathbb{Z}_{p}^{m}$ contains fewer than $p \cdot \operatorname{dim} \mathbf{H}$ points of $A$ (counting multiplicity).

Taking $\mathbf{H}=\mathbb{Z}_{p}^{m}$ one sees that every valid multiset has size at most $m p-1$. On the other hand, there are valid multisets of this size, see [3, Example 4.2]. Furthermore in case $m=2$ the definition of validity agrees with Definition 2 in the interesting case $|A| \leqslant 2 p-1$. Martin, Peilloux and Wong [3] made the following conjecture.

Conjecture 7. Let $p$ be an odd prime, let $m \geqslant 2$ be a positive integer, and let $A$ be a valid multiset contained in $\mathbb{Z}_{p}^{m}$ with $|A|=q p+k$, where $q \geqslant 1$ and $0 \leqslant k \leqslant p-1$.

(a) If $0 \leqslant k \leqslant p-3$, then $\# \sum A \geqslant(k+2) p^{q}$;

(b) If $k=p-2$, then $\# \sum A \geqslant p^{q+1}-1$.

(c) If $k=p-1$, then $\# \sum A \geqslant p^{q+1}$.

Again the definition of validity is such that, assuming Conjecture 7 , it would be "wasteful" for a multiset to be non-valid. Also, if the conjecture is true, it gives the best possible lower bounds, see [3, Discussion after Conjecture 4.3].

Notice in particular the following special case of the conjecture.

Conjecture 8. Let $p$ be an odd prime, let $m$ be a positive integer, and let $A$ be a valid multiset contained in $\mathbb{Z}_{p}^{m}$ with $|A|=m p-1$. Then $\sum A=\mathbb{Z}_{p}^{m}$.

In Section 4 we will show that the methods used in the proof of Theorem 5 can be adapted to show the following theorem.

Theorem 9. Conjecture 8 implies Conjecture \%.

Hence a special case generalising Peng's result (Theorem 3) implies the whole conjecture. Peng has actually generalised his result to $\mathbb{Z}_{p}^{m}$ in [5] but he considers a much wider class of multisets than the valid sets here, so the result in [5] is not helpful here.

Let us close the introduction by discussing the additive basis conjecture of Jaeger, Linial, Payan and Tarsi [2]. We need the following definition from [1].

Definition 10. For a prime $p$ and a positive integer $m$, let $f(p, m)$ denote the minimal integer $t$ such that, for any $t$ bases $B_{1}, \ldots, B_{t}$ of $\mathbb{Z}_{p}^{m}$ one has

$$
\sum\left(\bigcup_{i=1}^{t} B_{i}\right)=\mathbb{Z}_{p}^{m},
$$

where the union is let to be a multiset. 
For instance by splitting the set $A$ of size $2 p-2$ below Conjecture 4 into $p-1$ bases of $\mathbb{Z}_{p}^{2}$, one sees that for $p \geqslant 3$ and $m \geqslant 2, f(p, m) \geqslant p$. Jaeger, Linial, Payan and Tarsi [2] conjectured that $f(p, m)$ can be bounded from above by a function of $p$ alone and suggested that perhaps even $f(p, m)=p$. They showed that the conjecture has implications to group connectivity of graphs. Alon, Linial and Meshulam [1] showed that $f(p, m) \leqslant(p-1) \log m+p-2$, a bound which depends mildly on $m$.

We make the following related conjecture.

Conjecture 11. If $B_{1}, B_{2}, \ldots, B_{p-1}$ are bases of $\mathbb{Z}_{p}^{m}$ and $A \subset \mathbb{Z}_{p}^{m}$ is a (linearly) independent set of size $m-1$, then

$$
\sum\left(A \cup \bigcup_{i=1}^{p-1} B_{i}\right)=\mathbb{Z}_{p}^{m},
$$

where these unions are as multisets.

Clearly this conjecture in particular implies $f(p, m) \leqslant p$, so that the following theorem which we will prove in Section 4 shows that the conjecture of Martin, Peilloux and Wong actually implies the strongest possible form of the additive basis conjecture.

Theorem 12. Conjecture 11 is equivalent to Conjecture 8.

\section{Auxiliary results}

As in [3], we will take advantage of direct sum representations of $\mathbb{Z}_{p}^{m}$. Recall that a group $\mathbf{G}$ is an internal direct sum of subgroups $\mathbf{H}$ and $\mathbf{K}$ iff $\mathbf{H} \cap \mathbf{K}=\{e\}$ and $\mathbf{H}+\mathbf{K}=\mathbf{G}$. As usual, we write in this case $\mathbf{G}=\mathbf{H} \oplus \mathbf{K}$. In particular there exists a projection homomorphism $\pi_{\mathbf{H}}: \mathbf{G} \rightarrow \mathbf{H}$ that is the identity in $\mathbf{H}$ and vanishes in $\mathbf{K}$.

The following lemma shows that one can deduce information about $\# \sum A$ by studying a subgroup and a projection.

Lemma 13. Let $\mathbf{G}=\mathbf{H} \oplus \mathbf{K}$, and let $C$ be a multiset contained in $\mathbf{G}$. Let $D=C \cap \mathbf{H}$, let $F=C \backslash D$, and let $E=\pi_{\mathbf{K}}(F)$. Then

$$
\# \sum C \geqslant \# \sum D \cdot \# \sum E \text {. }
$$

Proof. This is [3, Lemma 2.8], but we give a short proof for completeness. Let $y \in \sum E$. Then by definition of $E, x+y \in \sum F$ for some $x \in \mathbf{H}$. Furthermore

$$
x+y+\sum D \subseteq(x+y+\mathbf{H}) \cap\left(\sum F+\sum D\right)=(y+\mathbf{H}) \cap \sum C .
$$

Hence, for each $y \in \sum E \subseteq \mathbf{K}$, the coset $y+\mathbf{H}$ contains at least $\# \sum D$ points of $\sum C$, and the claim follows since these cosets are disjoint.

Let us now cite Theorem 3 as Peng states and proves it (see [4, Theorem 2]) as it actually tells us something about non-valid sets as well. 
Lemma 14. Let $p \in \mathbb{P}$ and let $A$ be a multiset of size $2 p-1$ contained in $\mathbb{Z}_{p}^{2}$. Assume that $0 \notin A$ and each non-trivial subgroup of $\mathbb{Z}_{p}^{2}$ contains at most $p$ elements of $A$. Then $\sum A=\mathbb{Z}_{p}^{2}$.

Actually Lemma 14 is no stronger than Theorem 3 but follows from it, see Lemma 18. Lemma 14 lets us prove the case $k=p-2$ of Conjecture 4 easily.

Lemma 15. Let $p \in \mathbb{P}$ and let $A$ be a valid multiset contained in $\mathbb{Z}_{p}^{2}$ with $|A|=2 p-2$. Then $\# \sum A \geqslant p^{2}-1$.

Proof. Assume, contrary to the claim, that there are two distinct points $z, w \in \mathbb{Z}_{p}^{2} \backslash \sum A$. Let $B$ be the multiset $A$ joined by $z-w$. This multiset satisfies the hypothesis of Lemma 14 but $z \notin \sum A+\{0, z-w\}=\sum B$, a contradiction.

The following simple lemma will be the main tool in our inductive argument.

Lemma 16. Let $\mathbf{G}$ be an abelian group and let $A \subseteq \mathbf{G}$. Then for every $m \geqslant 2$,

$$
\#(A+\{0, z, 2 z, \ldots, m z\})-\#(A+\{0, z\}) \leqslant(m-1)(\#(A+\{0, z\})-\# A) .
$$

Proof. Here

$$
\begin{aligned}
\#(A+\{0, z, 2 z, \ldots, m z\}) & =\#\left(\bigcup_{i=0}^{m}(A+i z)\right) \\
& =\#\left(A \cup \bigcup_{i=1}^{m}((A+i z) \backslash(A+(i-1) z))\right) \\
& \leqslant \# A+\sum_{i=1}^{m} \#((A+i z) \backslash(A+(i-1) z)) \\
& =\# A+m \cdot \#((A+z) \backslash A),
\end{aligned}
$$

and the claim follows after a rearrangement.

For the proof of Theorem 12 we need the following direct consequence of the matroid union theorem (see for instance [7, Theorem 2 in Section 8.4]).

Lemma 17. Let $V$ be a vector space and let $A$ be a multiset contained in $V$. If $|U \cap A| \leqslant$ $k \cdot \operatorname{dim} U$ for every subspace $U \leqslant V$, then $A$ may be partitioned into $k$ sets $A_{1}, \ldots, A_{k}$ where every $A_{i}$ is linearly independent.

\section{Proof of Theorem 5}

Let $A$ be a valid multiset of size $p+k$ contained in $\mathbb{Z}_{p}^{2}$. As the case $k=p-2$ was handled in Lemma 15, we can assume that $0 \leqslant k \leqslant p-3$. For $z \in A$, write $A_{z}=A \cap\langle z\rangle$ and 
$A_{z}^{\mathrm{c}}=A \backslash A_{z}$. We will induct on $k$ but let us first handle the case $\left|A_{z}\right| \geqslant k+1$ for some $z \in A$ as in [3]. In this case $\left|A_{z}^{\mathrm{c}}\right|=|A|-\left|A_{z}\right| \leqslant p-1$, and by Lemmas 13 and 1

$$
\# \sum A \geqslant\left(\left|A_{z}\right|+1\right)\left(\left|A_{z}^{\mathrm{c}}\right|+1\right)=\left(\left|A_{z}\right|+1\right)\left(|A|-\left|A_{z}\right|+1\right)=\left|A_{z}\right|\left(|A|-\left|A_{z}\right|\right)+|A|+1
$$

which attains its minimum when $\left|A_{z}\right|$ is minimal or maximal. For both $\left|A_{z}\right|=k+1$ and $\left|A_{z}\right|=p-1$, the right hand side is $(k+2) p$ and the claim follows.

Hence we can assume from now on that, for every $z \in A,\left|A_{z}\right| \leqslant k$. Notice that as in [3] this in particular resolves the case $k=0$.

At this point our proof diverges from that in [3], where the authors modified the set $A$ to contain more elements in some subgroup by replacing $2 l$ points $x_{i}, z-x_{i} \in A, i=1, \ldots, l$ by $l$ copies of $z$. Here we instead set up an induction on $k$ (recall that $|A|=p+k$ ). As we already handled the case $k=0$, we can proceed directly to the induction step.

Assume, contrary to the claim, that $\# \sum A \leqslant(k+2) p-1$. Notice that, for every $z \in A$,

$$
\sum A=\sum(A \backslash\{z\})+\{0, z\}
$$

and here by the induction hypothesis $\# \sum(A \backslash\{z\}) \geqslant(k+1) p$. Hence

$$
\#\left(\sum(A \backslash\{z\})+\{0, z\}\right)-\# \sum(A \backslash\{z\}) \leqslant(k+2) p-1-(k+1) p=p-1 .
$$

Let $B$ be the multiset which consists of $A$ and $p-k-2$ additional copies of $z$, so that $|B|=2 p-2$. Since $|A \cap\langle z\rangle| \leqslant k, B$ is valid, so that by Lemma 15 , $\# \sum B \geqslant p^{2}-1$. On the other hand, applying Lemma 16 and recalling (1), one gets

$$
\begin{aligned}
\# \sum B= & \#\left(\sum(A \backslash\{z\})+\{0, z, 2 z, \ldots,(p-k-1) z\}\right) \\
\leqslant & \left(\# \sum(A \backslash\{z\})+\{0, z\}\right) \\
& \quad+(p-k-2)\left(\#\left(\sum(A \backslash\{z\})+\{0, z\}\right)-\# \sum(A \backslash\{z\})\right) \\
\leqslant & \# \sum A+(p-k-2)(p-1) \leqslant(k+2) p-1+(p-k-2)(p-1) \\
= & p^{2}-p+k+1 \leqslant p^{2}-2
\end{aligned}
$$

since $k \leqslant p-3$. Hence we have arrived to a contradiction so one must indeed have $\# \sum A \geqslant(k+2) p$.

\section{Proofs of Theorems 9 and 12}

To prove Theorem 9, we need a few lemmas. The first lemma shows that a stronger statement follows from Conjecture 8, in particular Lemma 14 follows from Theorem 3.

Lemma 18. Conjecture 8 implies the following: Let $p$ be an odd prime and let $m$ be $a$ positive integer. Let $A$ be a multiset contained in $\mathbb{Z}_{p}^{m}$ for which

$$
|A \cap \mathbf{H}| \leqslant p \operatorname{dim} \mathbf{H}
$$

for every subgroup $\mathbf{H} \leqslant \mathbb{Z}_{p}^{m}$. If $|A| \geqslant m p-1$, then $\sum A=\mathbb{Z}_{p}^{m}$. 
Proof. Let us induct on $m$. Case $m=1$ follows from Lemma 1 , so we can move to the induction step. We can clearly assume that $|A|=m p-1$. Let $\mathbf{H}$ be a maximal subgroup of $\mathbb{Z}_{p}^{m}$ for which equality holds in (2) (possibly $\mathbf{H}=\{0\}$ ), and write $\mathbb{Z}_{p}^{m}=\mathbf{H} \oplus \mathbf{K}$. If $\pi_{\mathbf{K}}(A \backslash \mathbf{H})$ were not a valid multiset, there would exist a non-trivial subgroup $K_{1} \leqslant K$ such that $\left|(A \backslash \mathbf{H}) \cap\left(\mathbf{H} \oplus \mathbf{K}_{\mathbf{1}}\right)\right| \geqslant p \cdot \operatorname{dim} \mathbf{K}_{\mathbf{1}}$ and consequently

$$
\begin{aligned}
A \cap\left(\mathbf{H} \oplus \mathbf{K}_{\mathbf{1}}\right) & =|A \cap \mathbf{H}|+\left|(A \backslash \mathbf{H}) \cap\left(\mathbf{H} \oplus \mathbf{K}_{\mathbf{1}}\right)\right| \\
& \geqslant p \cdot\left(\operatorname{dim} \mathbf{H}+\operatorname{dim} \mathbf{K}_{\mathbf{1}}\right)=p \cdot\left(\operatorname{dim} \mathbf{H} \oplus \mathbf{K}_{\mathbf{1}}\right)
\end{aligned}
$$

which contradicts the maximality of $\mathbf{H}$.

Hence $\pi_{\mathbf{K}}(A \backslash \mathbf{H})$ is a valid multiset contained in $\mathbf{K}$ with size

$$
|A|-|A \cap \mathbf{H}|=m p-1-p \cdot \operatorname{dim} \mathbf{H}=p \cdot \operatorname{dim} \mathbf{K}-1,
$$

so that $\sum \pi_{\mathbf{K}}(A \backslash \mathbf{H})=\mathbf{K}$ by the assumed Conjecture 8. Furthermore $A \cap \mathbf{H}$ has size $p \cdot \operatorname{dim} \mathbf{H}$ and dimension smaller than $m$, and thus by induction hypothesis $\sum(A \cap \mathbf{H})=\mathbf{H}$, and the claim follows from Lemma 13.

Theorem 12 follows now immediately:

Proof of Theorem 12. Conjecture 8 implies Conjecture 11 by Lemma 18 and Conjecture 11 implies Conjecture 8 by Lemma 17.

The following lemma follows from the previous lemma as Lemma 15 follows from Lemma 14.

Lemma 19. Conjecture 8 implies the following: Let $p$ be an odd prime, let $m$ be a positive integer, and let $A$ be a valid multiset contained in $\mathbb{Z}_{p}^{m}$ with $|A|=m p-2$. Then $\# \sum A \geqslant p^{m}-1$.

The third and fourth lemmas will let us show that we can assume that our multiset $A$ is not too concentrated in any subgroup (recall that also in the proof of Theorem 5 we first showed that we can assume that $|A \cap\langle z\rangle| \leqslant k$ for every $z \in A$ ).

Lemma 20. Let $m \geqslant 2$ and $\mathbb{Z}_{p}^{m}=\mathbf{H} \oplus \mathbf{K}$, where $0<\operatorname{dim} \mathbf{H}<m$. If $A$ is a valid multiset contained in $\mathbb{Z}_{p}^{m}$ with

$$
|A \backslash \mathbf{H}| \leqslant p \cdot \operatorname{dim} \mathbf{K}-1,
$$

then there exists a non-trivial subgroup $\mathbf{K}^{\prime} \lesseqgtr \mathbb{Z}_{p}^{m}$ such that, writing $\mathbb{Z}_{p}^{m}=\mathbf{H}^{\prime} \oplus \mathbf{K}^{\prime}$, $\pi_{\mathbf{K}^{\prime}}\left(A \backslash \mathbf{H}^{\prime}\right)$ is a valid multiset contained in $\mathbf{K}^{\prime}$.

Proof. If $\pi_{\mathbf{K}}(A \backslash \mathbf{H})$ is valid, the claim follows immediately. Otherwise there is a non-trivial subgroup $\mathbf{K}_{1} \leqslant \mathbf{K}$ such that

$$
\left|(A \backslash \mathbf{H}) \cap\left(\mathbf{H} \oplus \mathbf{K}_{1}\right)\right| \geqslant p \cdot \operatorname{dim} \mathbf{K}_{1} .
$$

Let $\mathbf{K}_{1}$ be maximal such subgroup and $\mathbf{K}=\mathbf{K}_{1} \oplus \mathbf{K}_{2}$. The bounds (4) and (3) together imply that $\mathbf{K}_{1} \lesseqgtr \mathbf{K}$ so that $\mathbf{K}_{2} \neq\{0\}$. 
If $\pi_{\mathbf{K}_{2}}\left(A \backslash\left(\mathbf{H} \oplus \mathbf{K}_{1}\right)\right)$ is valid, the claim follows with $\mathbf{K}^{\prime}=\mathbf{K}_{2}$ and $\mathbf{H}^{\prime}=\mathbf{H} \oplus \mathbf{K}_{1}$. Otherwise there exists a non-trivial subgroup $\mathbf{K}_{3} \leqslant \mathbf{K}_{2}$ such that

$$
\left|\left(A \backslash\left(\mathbf{H} \oplus \mathbf{K}_{1}\right)\right) \cap\left(\mathbf{H} \oplus \mathbf{K}_{1} \oplus \mathbf{K}_{3}\right)\right| \geqslant p \cdot \operatorname{dim} \mathbf{K}_{3} .
$$

Combining with (4) gives

$$
\left|(A \backslash \mathbf{H}) \cap\left(\mathbf{H} \oplus \mathbf{K}_{1} \oplus \mathbf{K}_{3}\right)\right| \geqslant p \cdot\left(\operatorname{dim} \mathbf{K}_{1}+\operatorname{dim} \mathbf{K}_{3}\right)=p \cdot \operatorname{dim}\left(\mathbf{K}_{1} \oplus \mathbf{K}_{3}\right)
$$

which contradicts the maximality of $\mathbf{K}_{1}$.

Lemma 21. Let $p$ be an odd prime and define $f: \mathbb{Z}_{\geqslant 0} \rightarrow \mathbb{N}$ by putting for each $q \geqslant 0$ and $0 \leqslant k \leqslant p-1$,

$$
f(q p+k)= \begin{cases}k+1 & \text { if } q=0 \text { and } 0 \leqslant k \leqslant p-1 \\ (k+2) p^{q} & \text { if } q \geqslant 1 \text { and } 0 \leqslant k \leqslant p-3 \\ p^{q+1}-1 & \text { if } q \geqslant 0 \text { and } k=p-2 \\ p^{q+1} & \text { if } q \geqslant 0 \text { and } k=p-1 ;\end{cases}
$$

Then for every $n_{1}, n_{2} \in \mathbb{Z}_{\geqslant 0}$ one has $f\left(n_{1}\right) \cdot f\left(n_{2}\right) \geqslant f\left(n_{1}+n_{2}\right)$.

Proof. Write $n_{i}=q_{i} p+k_{i}$. First note that

$f\left(q_{1} p+p-2\right) f(p-2)=\left(p^{q_{1}+1}-1\right)(p-1) \geqslant(p-2)\left(p^{q_{1}+1}-1\right)=f\left(q_{1} p+p-2+p-2\right)$, so we can assume that if $k_{1}=k_{2}=p-2$ then $q_{2} \neq 0$. One has

$$
\frac{f(q p+k)}{f(q p+k-1)}= \begin{cases}\frac{k+1}{k}=1+\frac{1}{k} & \text { if } q=0 \text { and } 0<k \leqslant p-1 \\ \frac{k+2}{k+1}=1+\frac{1}{k+1} & \text { if } q \geqslant 1 \text { and } 0 \leqslant k \leqslant p-3 \\ \frac{p^{q+1}-1}{p^{q}(p-1)}=1+\frac{p^{q}-1}{p^{q}(p-1)} & \text { if } q \geqslant 1 \text { and } k=p-2 ; \\ \frac{p^{q+1}}{p^{q+1}-1}=1+\frac{1}{p^{q+1}-1} & \text { if } q \geqslant 0 \text { and } k=p-1 .\end{cases}
$$

From this we see that for every $q_{1}, q_{2} \geqslant 0$ and $0 \leqslant k_{1} \leqslant k_{2} \leqslant p-2$ (with $q_{1} p+k_{1}>0$ and not $\left.\left(k_{1}, k_{2}, q_{2}\right)=(p-2, p-2,0)\right)$ one has

$$
\begin{aligned}
\frac{f\left(q_{1} p+k_{1}\right)}{f\left(q_{1} p+k_{1}-1\right)} & \geqslant \frac{f\left(q_{2} p+k_{2}+1\right)}{f\left(q_{2} p+k_{2}\right)} \\
\Longleftrightarrow f\left(q_{1} p+k_{1}\right) f\left(q_{2} p+k_{2}\right) & \geqslant f\left(q_{1} p+k_{1}-1\right) f\left(q_{2} p+k_{2}+1\right) .
\end{aligned}
$$

Applying (5) repeatedly to $f\left(n_{1}\right) f\left(n_{2}\right)$, we can assume that either $k_{1}=p-1$ or $k_{2}=p-1$, and consequently, by symmetry, that $k_{1}=p-1$. The proof can then be completed by an easy case-by-case check according to the value of $k_{2}$. 
Proof of Theorem 9. Let $f$ be as in Lemma 21. Conjecture 7 is equivalent to the claim that for every $m \geqslant 1$ and any valid multiset $A$ contained in $\mathbb{Z}_{p}^{m}$ one has $\# \sum A \geqslant f(|A|)$ (since the latter claim holds if $m=1$ or if $|A|<p$ by Lemmas 1 and 13).

Let us induct on $m$. Lemma 1 takes care of the case $m=1$, so we can move to the induction step. Let $|A|=q p+k$. We will induct also on $k$ but let us first consider the case that for some non-trivial subgroup $\mathbf{H} \lesseqgtr \mathbb{Z}_{p}^{m}$ one has $|A \backslash \mathbf{H}| \leqslant p \cdot(m-\operatorname{dim} \mathbf{H})-1$. In this case Lemma 20 implies that there exists a non-trivial subgroup $\mathbf{K}^{\prime} \lesseqgtr \mathbb{Z}_{p}^{m}$ such that, writing $\mathbb{Z}_{p}^{m}=\mathbf{H}^{\prime} \oplus \mathbf{K}^{\prime}, \pi_{\mathbf{K}^{\prime}}\left(A \backslash \mathbf{H}^{\prime}\right)$ is a valid multiset contained in $\mathbf{K}^{\prime}$. Since $\operatorname{dim} \mathbf{H}^{\prime}, \operatorname{dim} \mathbf{K}^{\prime}<m$, by the induction hypothesis

$$
\# \sum \pi_{\mathbf{K}^{\prime}}\left(A \backslash \mathbf{H}^{\prime}\right) \geqslant f\left(\left|A \backslash \mathbf{H}^{\prime}\right|\right) \text { and } \# \sum\left(A \cap \mathbf{H}^{\prime}\right) \geqslant f\left(\left|A \cap \mathbf{H}^{\prime}\right|\right) .
$$

Hence by Lemmas 13 and 21

$$
\# \sum A \geqslant f\left(\left|A \backslash \mathbf{H}^{\prime}\right|\right) \cdot f\left(\left|A \cap \mathbf{H}^{\prime}\right|\right) \geqslant f(|A|)
$$

and the claim follows.

Thus we can assume that

$$
|A \backslash \mathbf{H}| \geqslant p \cdot(m-\operatorname{dim} \mathbf{H})
$$

for every non-trivial subgroup $\mathbf{H} \lesseqgtr \mathbb{Z}_{p}^{m}$. In particular taking $\mathbf{H}=\langle z\rangle$ for some $z \in \mathbb{Z}_{p}^{m}$, we see that we can assume that $q=m-1$, so that $|A|=(m-1) p+k$. By this and (6) we can thus assume that for every subgroup $\mathbf{H} \leqslant \mathbb{Z}_{p}^{m}$ one has

$$
|A \cap \mathbf{H}|=|A|-|A \backslash \mathbf{H}| \leqslant(m-1) p+k-p \cdot(m-\operatorname{dim} \mathbf{H})=p \cdot(\operatorname{dim} \mathbf{H}-1)+k .
$$

Taking here $\mathbf{H}=\langle z\rangle$ for some $z \in A$, we see that we can assume that $k>0$. On the other hand, Lemma 19 lets us assume that $k \leqslant p-3$.

From now on the proof proceeds almost exactly as the proof of Theorem 5 , so let us induct also on $k$ and assume, contrary to the claim, that $\# \sum A \leqslant(k+2) p^{m-1}-1$. Recall that, for every $z \in A$,

$$
\sum A=\sum(A \backslash\{z\})+\{0, z\},
$$

and here by the induction hypothesis $\# \sum(A \backslash\{z\}) \geqslant(k+1) p^{m-1}$. Hence

$$
\#\left(\sum(A \backslash\{z\})+\{0, z\}\right)-\# \sum(A \backslash\{z\}) \leqslant(k+2) p^{m-1}-1-(k+1) p^{m-1}=p^{m-1}-1 .
$$

Let $B$ be the multiset which consists of $A$ and $p-k-2$ additional copies of $z$, so that $|B|=m p-2$. Since (7) holds for every non-trivial subgroup $\mathbf{H}, B$ is valid, so that, by Lemma 19, $\# \sum B \geqslant p^{m}-1$. On the other hand, applying Lemma 16 recalling (8), one gets

$$
\begin{aligned}
\# \sum B & =\#\left(\sum(A \backslash\{z\})+\{0, z, 2 z, \ldots,(p-k-1) z\}\right) \\
& \leqslant \# \sum A+(p-k-2)\left(\# \sum A-\# \sum(A \backslash\{z\})\right) \\
& \leqslant(k+2) p^{m-1}-1+(p-k-2)\left(p^{m-1}-1\right)=p^{m}-p+k+1 \leqslant p^{m}-2
\end{aligned}
$$

since $k \leqslant p-3$. 
The proof actually tells us that if, for some $M \geqslant 2$, Conjecture 8 holds for every $m \leqslant M$, then so does Conjecture 7. In particular, as was shown already in Section 3, Theorem 3 implies Theorem 5.

\section{Acknowledgements}

The author would like to thank the referee for useful comments, in particular for suggesting the addition of Theorem 12 (and related Conjecture 11 and Lemma 17).

\section{References}

[1] N. Alon, N. Linial, and R. Meshulam. Additive bases of vector spaces over prime fields. J. Combin. Theory Ser. A, 57(2):203-210, 1991.

[2] F. Jaeger, N. Linial, C. Payan, and M. Tarsi. Group connectivity of graphs - a nonhomogeneous analogue of nowhere-zero flow properties. J. Combin. Theory Ser. B, 56(2):165-182, 1992.

[3] G. Martin, A. Peilloux, and E. B. Wong. Lower bounds for sumsets of multisets in $\mathbb{Z}_{p}^{2}$. Integers, to appear, pre-print available at arXiv:1107.4392v3.

[4] C. Peng. Addition theorems in elementary abelian groups. I. J. Number Theory, 27:46-57, 1987.

[5] C. Peng. Addition theorems in elementary abelian groups. II. J. Number Theory, 27:58-62, 1987.

[6] T. Tao and V. H. Vu. Additive combinatorics, volume 105 of Cambridge Studies in Advanced Mathematics. Cambridge University Press, Cambridge, paperback edition, 2010.

[7] D. J. A. Welsh. Matroid Theory. L.M.S. Monographs. Academic Press Inc. Ltd., London, 1976. 